(C) 2012 IEEE. Personal use of this material is permitted. Permission from IEEE must be obtained for all other uses, in any current or future media, including reprinting/republishing this material for advertising or promotional purposes, creating new collective works, for resale or redistribution to servers or lists, or reuse of any copyrighted component of this work in other works. 


\section{RSS-based Indoor Positioning Accuracy Improvement Using Antenna Array in WLAN Environments}

\author{
Gayan Attanayake \\ Dept. Electrical and Computer Engineering \\ Curtin University \\ Bentley, WA, Australia \\ Email: a.attanayake@postgrad.curtin.edu.au
}

\author{
Yue Rong \\ Dept. Electrical and Computer Engineering \\ Curtin University \\ Bentley, WA, Australia \\ Email: y.rong@curtin.edu.au
}

\begin{abstract}
The interest in utilizing Wi-Fi signals for indoor location estimation purposes has been increased recently due to wide deployment of WLANs. Received signal strength (RSS) based approach has become an attractive candidate for positioning owing to its simplicity and low-complexity, which can be easily implemented in modern wireless devices such as laptops and PDAs. However, the challenging nature of indoor wireless propagation environments provoke time varying location estimations from RSS based positioning algorithms. In this paper, we have shown that this variability of the location estimations can be reduced by introducing an antenna array at the receiving station.

In our proposed approach, the variation of the received signal power with respect to time is averaged using a uniform linear antenna array (ULA) at the mobile station. We further explore the impact of number of array elements on the accuracy of the position estimations by using representative set of multilateration algorithms. In the first phase of analysis, we consider uncorrelated Rayleigh fading channels on each antenna element whilst in the second phase, we take into account the fading correlation between antenna elements using the spatial correlation function for two-dimensional (2D) diffuse field. The proposed positioning technique can be integrated into IEEE 802.11 compatible receivers with single-input multiple-output (SIMO) capability, thus be able to use for robust indoor localization purposes.

Index Terms-Indoor Positioning; Antenna Array; RSS; Fading Correlation; Lateration.
\end{abstract}

\section{INTRODUCTION}

Indoor localization has recently been of significant interest in wireless networks owing to the requirement of accurate location base services (LBSs) for diverse of new applications, such as health care monitoring, personal tracking, inventory control and location specific information routing. Indoor environments, however, impose great challenge for accurate localization due to their inherent multipath effects including reflections and diffractions. These have made signal characterization hard with respect to location, thereby placing localization of wireless devices in indoors as an active area of research.

To date, various physical modalities have been used for indoor positioning including time of arrival (TOA) [1], time difference of arrival (TDOA) [2], angle of arrival (AOA) [3] and received signal strength (RSS) [4]. RSS-based techniques have drawn considerable attention since they allow the reuse of existing communication infrastructure, thus yield low cost, simple and feasible localization solution. However, RSS measurements are susceptible to short term fading. Consequently, it necessitates the need of complex algorithms for robust localization [1], which require substantial processing power and time. Utilization of an antenna array, with closely spaced antenna elements at the mobile station (MS), presents the opportunity to smooth out these fading effects and employ low complex positioning algorithms for accurate localization purposes whilst maintaining the same number of wireless access points (WAPs) used by localization system.

In this work, we analyse the variability reduction of RSS by employing multi-element antenna systems at MS. In order to relate our analysis for indoors, we consider Rayleigh-pluslog normal statistical model that successfully describes the RSS variation in open indoor environments [5]. Our analysis of employing antenna arrays is of two-fold. First, we consider totally uncorrelated channels on each antenna element. Second, we replace the uncorrelated channel model with spatial correlation function for 2D omni-directional diffuse field to model the effect of fading correlation between antenna elements, which appropriately reflects narrowband Rayleigh fading in fixed wireless systems [6]. We then investigate the variance reduction of RSS with respect to the number of antenna elements and inter-element spacing. Furthermore, we verify our analytical outcomes through simulations.

In next step, we numerically simulate the effect of using an antenna array at MS for location accuracy. We use two representative lateration algorithms: linear least square (LLS) that gives closed-form location estimations, and non-linear least square (NLS) that gives more accurate location estimations by solving an optimization problem [7]. Simulation results demonstrate that using antenna array at MS provides better location estimations compared to single antenna scenario. Moreover, the results show that accuracy improves with the number of elements on the antenna array.

The rest of this paper is organized as follows. In Sec- 
tion II, we first introduce the radio propagation model for fixed indoor wireless environments such as shopping malls and large halls. Second, we present the diversity combining technique employed at the receiving station. We then present the network operation of localization in wireless infrastructure. The analysis of RSS variance reduction in uncorrelated and correlated channel models, is also presented under this section. The positioning techniques used for simulations are described in Section III. In Section IV, we demonstrate some numerical examples, in particular, we emphasise the localization accuracy improvement with respect to the number of array elements. Finally, conclusions are drawn in Section V.

\section{SySTEM MODEL}

\section{A. RSS Model}

Although presenting a generic model for RSS is not feasible in indoor environments, it is well established that for open indoor areas, the signal statistics can be modelled as a combination of a quasi stationary process (i.e. multipath) superimposed on a large scale process (shadowing and pathloss) [5]. In our simulations, we assume that small scale fading follows a Rayleigh distribution [8]. First, we construct the multipath fading signal from in-phase and quadrature Gaussian noise samples, which are then filtered, so that the frequency domain of the output envelop has the desired Doppler spectrum for a indoor fixed wireless communication system [9]. Here, we use the bell-shaped spectrum in (1) as recommended by the IEEE 802.11 community, which models the indoor areas for WLAN applications where transmitter and receiver remain stationary while people are moving in between [10]

$$
D(f)=\frac{1}{1+\left(\frac{3 f}{f_{d}}\right)^{2}}
$$

Here, $f_{d}$ is the frequency at which the spectrum is dropped to $10 \%$ of its peak and it is approximately equal to $3 \mathrm{kHz}$ for $2.4 \mathrm{GHz}$ centre frequency systems according to [10]. The maximum frequency component of the Doppler spectrum $f_{\text {max }}$ is set arbitrarily to $5 f_{d}$ [9].

Second, we characterize the received power $(\mathrm{dBm})$ at a point which is $d_{i}$ distance away from a WAP by

$$
P_{r}\left(d_{i}\right)=P_{t}-P_{L}\left(d_{0}\right)-10 n_{p} \log _{10}\left(\frac{d_{i}}{d_{0}}\right)-M_{F_{i}}-X_{\sigma_{i}}
$$

where $P_{t}(\mathrm{dBm})$ is the WAP transmit power (typically $40 \mathrm{dBm}$ for Wi-Fi systems), $P_{L}\left(d_{0}\right)(\mathrm{dB})$ is the path-loss at Fraunhofer distance $\left(d_{0} \approx 1 \mathrm{~m}\right.$ for Wi-Fi systems according to [11]), $n_{p}$ is the path-loss gradient, $M_{F_{i}}$ represents the small-scale fading (dB) due to multipath propagation whilst $X_{\sigma_{i}}$ is the long-term fading $(\mathrm{dB})$ due to shadowing. However, for indoor environments such as large shopping malls and office floors with small desk partitions, $X_{\sigma_{i}}$ may be neglected. Nevertheless, the temporal fluctuation of received power at a given point in space is totally characterized by $M_{F_{i}}$ in (2).

\section{B. RSS Variance Reduction with Antenna Array}

In this subsection we investigate the result of using an antenna array at the receiving station to reduce the temporal fluctuation of received signal power. We consider a single user communication system and diversity receive channel model with maximal ratio combining (MRC). First, we assume that the receiver lies in a rich scattering environment described by the one-ring model in [6], also, in particular, a sufficiently large inter-element $\operatorname{space}(\geq \lambda / 2$, where $\lambda$ is the wavelength). Thus, the channels on each antenna elements can considered to be uncorrelated. Second, we remove the latter assumption and analyse the temporal fluctuation of the power against the inter-element spacing. Fig. 1 shows the system model for both of these investigations.

1) Uncorrelated Rayleigh Fading: Each channel from transmitter to receiver in Fig.1 are circularly symmetric complex Gaussian distributed, denoted by $h_{i} \sim \tilde{N}\left(0,2 \sigma^{2}\right)$, where $h_{i}=x_{I}+j x_{Q}$ in which $x_{I}$ and $x_{Q}$ are i.i.d $N\left(0, \sigma^{2}\right)$. When the channel is slow and flat fading, the received signal vector $\mathbf{z}$ can be written as

$$
\mathbf{z}=\mathbf{y} u(t)+\mathbf{n}
$$

where $\mathbf{z}=\left[z_{1}, z_{2}, \ldots, z_{L}\right]^{T}, \mathbf{y}=\left[y_{1}, y_{2}, \ldots, y_{L}\right]^{T}$ and $\mathbf{n}=\left[n_{1}, n_{2}, \ldots, n_{L}\right]^{T}, y_{i}$ is the channel between transmitter and $i^{t h}$ receiving antenna, $u(t)$ is the unit power transmitted signal and $n_{i}$ is the associated AWGN with the channel. According to the model illustrated in Fig. 1, the channels become uncorrelated when the channel covariance matrix $\mathbf{C}_{H}=\mathbf{I}_{L}$, where $\mathbf{I}_{L}$ is an $L \times L$ unity matrix, and $L$ is the number of elements in receiver antenna array. After diversity combining with MRC, the output signal is given by

$$
\begin{aligned}
r(t) & =\mathbf{w}^{H} \mathbf{y} u(t)+\mathbf{w}^{H} \mathbf{n} \\
& =\mathbf{w}^{H} \mathbf{I}_{L} \mathbf{h} u(t)+\mathbf{w}^{H} \mathbf{n} .
\end{aligned}
$$

To maximize the SNR, by Cauchy-Schwarz inequality, we take $\mathbf{w}=\kappa \mathbf{h}$, where $\kappa$ is an arbitrary constant [12]. Therefore, we can derive the effective power of the combiner's output by

$$
\begin{aligned}
P_{\text {eff }} & =\kappa^{2}\left|\mathbf{h}^{H} \mathbf{h}\right|^{2} \\
& =\kappa^{2}\left(\left|h_{1}\right|^{2}+\left|h_{2}\right|^{2}+\cdots+\left|h_{L}\right|^{2}\right)^{2}
\end{aligned}
$$

where the term $\left(\left|h_{1}\right|^{2}+\left|h_{2}\right|^{2}+\cdots+\left|h_{L}\right|^{2}\right)$ is chi-squared distributed with $2 L$ degrees of freedom. We consider $\sigma=1$ without loss of generality and choose $\kappa$ such that it normalizes the ensemble average, $\mathbb{E}\left\{P_{\text {eff }}\right\}=4 \kappa^{2} L(L+1)$. Thus, we get $\kappa=1 /(2 \sqrt{L(L+1)})$. Furthermore, the variance of $P_{\text {eff }}$ can be derived as

$$
\sigma_{P}^{2}=\frac{2}{L}\left(2+\frac{1}{(L+1)}\right)
$$

It is clear from (6) that the variance of the effective output power reduces with number of array elements. Moreover, Fig. 2 illustrates that a fade reduction of $21.335 \mathrm{~dB}$ with a dynamic range of $22.82 \mathrm{~dB}$ can be achieved by using four elements on the antenna array, while a fade reduction of $20.595 \mathrm{~dB}$ with a 


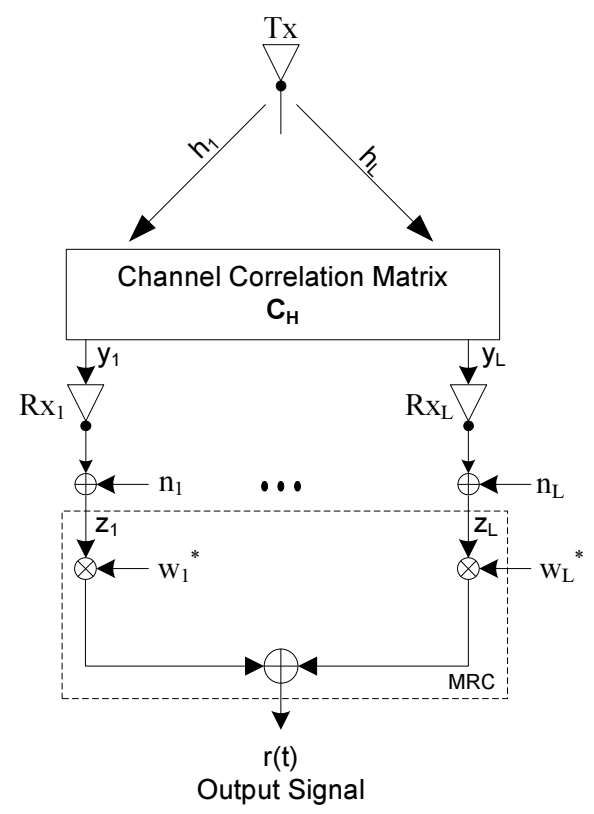

Figure 1. System Model

dynamic range of $13.056 \mathrm{~dB}$ can be achieved by using eight elements.

2) Correlated Rayleigh Fading: We previously assumed that the channels experience uncorrelated fading. However, in real propagation environments, channel fadings may not be independent owing, for example, to insufficient spacing between antenna elements [6]. In this subsection, we analyse the behaviour of the variance of the $P_{\text {eff }}$ with respect to interelement spacing. Here, we confine our analysis to two antenna elements and Rayleigh fading channels with no dominant component. We refer to the spatially uncorrelated $1 \times 2$ SIMO channel vector as $\mathbf{h}$. As illustrated in [13], for a $2 \mathrm{D}$ omni directional diffuse field, the channel covariance matrix become

$$
\mathbf{C}_{H}=\left[\begin{array}{cc}
1 & J_{0}\left(\frac{2 \pi}{\lambda} d\right) \\
J_{0}\left(\frac{2 \pi}{\lambda} d\right) & 1
\end{array}\right]
$$

and the correlated channel vector is given by [6]

$$
\mathbf{y}=\boldsymbol{\Phi}^{\frac{1}{2}} \mathbf{h}
$$

where $\boldsymbol{\Phi}^{\frac{1}{2}} \boldsymbol{\Phi}^{\frac{H}{2}}=\mathbf{C}_{H}, J_{0}($.$) is the zeroth order Bessel function$ of first kind, $\lambda$ is the wavelength, and $d$ is the inter-element space. From (7) we can obtain

$$
\boldsymbol{\Phi}=\left[\begin{array}{cc}
1 & 0 \\
J_{0}\left(\frac{2 \pi}{\lambda} d\right) & \sqrt{1-\left(J_{0}\left(\frac{2 \pi}{\lambda} d\right)\right)^{2}}
\end{array}\right] .
$$

The effective power output is given by

$$
\begin{aligned}
P_{\text {eff }} & =\kappa^{2}\left|\mathbf{y}^{H} \mathbf{y}\right|^{2} \\
& =\kappa^{2}\left(\mathbf{h}^{H} \boldsymbol{\Phi}^{\frac{H}{2}} \boldsymbol{\Phi}^{\frac{1}{2}} \mathbf{h}\right)^{2} .
\end{aligned}
$$

Similar to uncorrelated analysis, in order to normalize the ensemble average, $\mathbb{E}\left\{P_{\text {eff }}\right\}=8 \kappa^{2}$, we choose $\kappa=\frac{1}{2 \sqrt{2}}$.

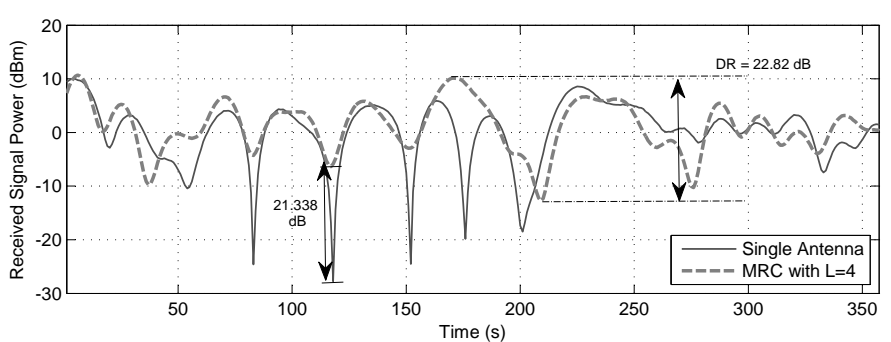

(a)

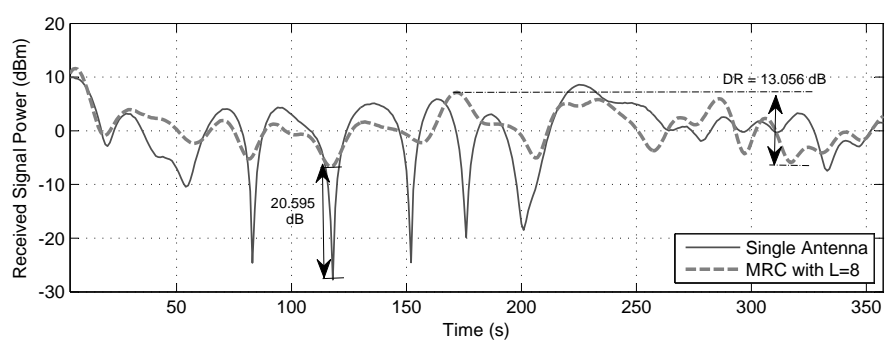

(b)

Figure 2. Received Signal Strength: (a) 4-element array (b) 8-element array

Furthermore, variance of $P_{\text {eff }}$ in (10) can be derived as

$$
\sigma_{P}^{2}=42\left(J_{0}(m d)\right)^{2}\left(1-\left(J_{0}(m d)\right)^{2}\right),
$$

where $m=(2 \pi / \lambda)$. Interestingly (11) reflects a sinc shape as depicted in Fig. 3 where the envelop of the $\sigma_{P}^{2}$ decreases as inter-element spacing increases. Thus, with a inter-element spacing $4.7 \mathrm{~cm}$ (in a $2.4 \mathrm{GHz}$ system), we can achieve a minimum variance for a two element MRC diversity reception system in a rich scatter Rayleigh fading environment. Moreover, we illustrate the effect of inter-element space on localization accuracy in Section IV.

\section{Network Operation for Localization}

In our proposed positioning prototype, we assume a clientbased localization engine, in which MS first scans the received signal power from three or more WAPs. This is called the training phase. Then MS estimate the distance from itself to each of corresponding WAPs. Finally, MS locates its position using one of the representative lateration algorithms which are discussed in next section. This is typically referred to run-time localization phase. The temporal variability of the position estimations can be mitigated using an antenna array at the receiving station.

\section{Proposed Algorithm}

In this section, we present two lateration based algorithms that are widely used for localization to validate our analysis. We study both non-linear least squares (NLS) and linear least squares (LLS) methods. Also we introduce the application of Armijo's rule [14] to effectively solve the NLS problem that results converged location estimations.

First, we present the notation for NLS and LLS, and, in turn, introduce the pertinent mathematical background for both 


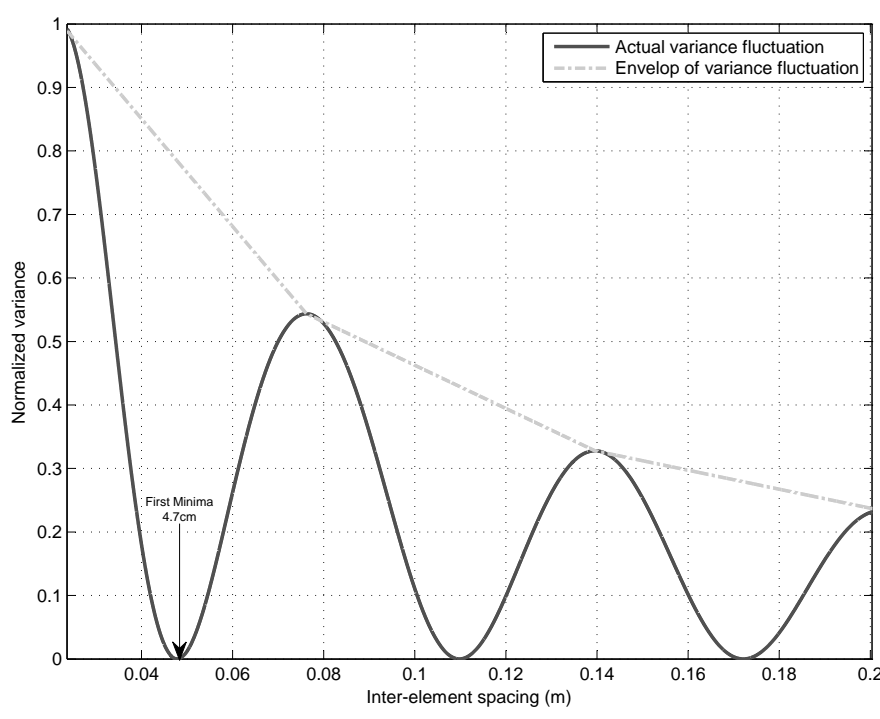

Figure 3. Variance fluctuation with inter-element spacing in a correlated twoelement antenna array

localization approaches. Since we consider a client-based localization approach, WAP and MS (i.e. client) always assume the roles of transmitter and receiver. We use the notation $\mathbf{s}=[x, y]^{T}$ to represent the variable location parameter vector. The estimated location of the MS is then referred to $\hat{\mathbf{s}}=[\hat{x}, \hat{y}]^{T}$. The observed received signal strength from $j^{t h}$ transmitter at the client is denoted by $P_{r j}(\mathrm{dBm})$. We define the RSS observation parameter $v_{j}(\mathrm{~dB})=P_{r j}\left(d_{0}\right)-P_{r j}$. Further, we denote the pathloss at a point $(x, y)$ by $L_{j}(\mathbf{s})(\mathrm{dB})=$ $P_{r j}\left(d_{0}\right)-P_{r j}(\mathbf{s})$. Here, $P_{r j}\left(d_{0}\right)$ and $P_{r j}(\mathbf{s})$ refer to the received signal levels from $j^{\text {th }}$ transmitter at the Fraunhofer distance and a point $(x, y)$ respectively.

\section{A. Non-linear Least Squares (NLS)}

Given the RSS observation vectors and relative pathlosses at a point $(x, y)$ with respect to $M$ number of WAPs, the location of the wireless node can be estimated by finding $\hat{\mathbf{s}}$ satisfying

$$
\hat{\mathbf{s}}=\underset{\mathbf{s}}{\operatorname{argmin}}\left\{\varepsilon(\mathbf{s})=\sum_{j=1}^{M} r_{j}^{2}(\mathbf{s})\right\}
$$

where the residual $r_{j}(\mathbf{s})=v_{j}-L_{j}(\mathbf{s})$. In order to solve the optimization problem in (12) we deploy widely used gradient descent algorithm with $(k+1)^{t h}$ iteration given by

$$
\mathbf{s}_{k+1}=\mathbf{s}_{k}+\mu_{k} \mathbf{D}_{k},
$$

where $\mu_{k}$ is the step size, $\mathbf{D}_{k}=-\nabla \varepsilon\left(\mathbf{s}_{k}\right)$ and $\mathbf{s}_{k}=\left[x_{k}, y_{k}\right]^{T}$. A suitable step size for $(k+1)^{t h}$ step can be found by using Armijo's rule. From (12), the gradient can be derived as

$$
\nabla \varepsilon\left(\mathbf{s}_{k}\right)=\frac{-20 n_{p}}{\ln 10}\left[\begin{array}{c}
\sum_{j=1}^{M} r_{j}\left(\mathbf{s}_{k}\right) \\
\sum_{j=1}^{M} r_{j}\left(\mathbf{s}_{k}\right)\left(\frac{\left(x_{k}-x_{j}\right)}{\left(x_{k}-x_{j}\right)^{2}+\left(y_{k}-y_{j}\right)^{2}}\right) \\
\left(\frac{\left(y_{k}-y_{j}\right)}{\left(x_{k}-x_{j}\right)^{2}+\left(y_{k}-y_{j}\right)^{2}}\right)
\end{array}\right] .
$$

Define the convex function $f\left(\mu_{k}\right)=\varepsilon\left(\mathbf{s}_{k+1}\right)=\varepsilon\left(\mathbf{s}_{k}+\mu_{k} \mathbf{D}_{k}\right)$. The estimate of convex function can be chosen such that $\hat{f}\left(\mu_{k}\right)=\varepsilon\left(\mathbf{s}_{k}\right)-\mu_{k} \epsilon \nabla \varepsilon^{T}\left(\mathbf{s}_{k}\right) \nabla \varepsilon\left(\mathbf{s}_{k}\right)$, where $0<\epsilon<1$. The step sizes for each iteration can be decided as for [14];

- Step 0: $\mu_{0}=\bar{\mu}>0$; set $k=0$.

- Step $k$ : if $f\left(\mu_{k}\right) \leq \hat{f}\left(\mu_{k}\right)$, choose $\mu_{k}$ as the step size and stop. Otherwise, take $\mu_{k+1}=\frac{1}{\alpha} \mu_{k}$, where $\alpha$ is chosen such that step size would not be too small (in our simulations we use $\alpha=2$ ).

However, due to considerable iterations for accurate estimations, the NLS approach is relatively high in computational complexity.

\section{B. Linear Least Squares (LLS)}

LLS approach linearises the NLS problem by introducing constraints in the formulation, and obtain a closed-form solution of location estimate [7]. On the other hand, LLS has got less computational complexity. From the RSS observations at $j^{\text {th }}$ WAP, we can obtain the range estimate $\hat{d}_{j}=d_{0} 10^{v_{j} /\left(10 n_{p}\right)}$, where $\hat{d}_{j}$ is the estimated distance to MS from $j^{t h}$ WAP. Furthermore, geometrically, a point $(x, y)$ on the perimeter of a circle with radius $\hat{d}_{j}$, and center $\left(x_{j}, y_{j}\right)$ satisfies

$$
\hat{d}_{j}^{2}=\left(x-x_{j}\right)^{2}+\left(y-y_{j}\right)^{2} .
$$

Considering the distance estimations made at MS for access points 1 and $i$, and from (13), we can write

$$
\left(x_{i}-x_{1}\right) x+\left(y_{i}-y_{1}\right) y=\frac{1}{2}\left(\left(x_{i}^{2}+y_{i}^{2}\right)-\left(x_{1}^{2}+y_{1}^{2}\right)-\left(\hat{d}_{i}^{2}-\hat{d}_{1}^{2}\right)\right) \text {. }
$$

Now, with observations made from $M(\geq 3)$ number of access points with estimated radii $\hat{d}_{i}$ and centers $\left(x_{i}, y_{i}\right)$, for $i=$ $2, \ldots, M$, we can derive

$$
\mathbf{b}=\mathbf{A} \mathbf{s} .
$$

where

$$
\mathbf{A}=\left[\begin{array}{cccc}
\left(x_{2}-x_{1}\right) & \left(x_{3}-x_{1}\right) & \cdots & \left(x_{M}-x_{1}\right) \\
\left(y_{2}-y_{1}\right) & \left(y_{3}-y_{1}\right) & \cdots & \left(y_{M}-y_{1}\right)
\end{array}\right]^{T}
$$

and

$$
\mathbf{b}=\frac{1}{2}\left[\begin{array}{c}
\left|\mathbf{s}_{2}\right|^{2}-\left|\mathbf{s}_{1}\right|^{2}-\left({\hat{d_{2}}}^{2}-{\hat{d_{1}}}^{2}\right) \\
\vdots \\
\left|\mathbf{s}_{M}\right|^{2}-\left|\mathbf{s}_{1}\right|^{2}-\left({\hat{d_{M}}}^{2}-{\hat{d_{1}}}^{2}\right)
\end{array}\right]
$$

Here $\mathbf{s}_{i}=\left[x_{i}, y_{i}\right]^{T}$, is the location vector of $i^{t h}$ access point with $\left|\mathbf{s}_{i}\right|^{2}=x_{i}^{2}+y_{i}^{2}, i=1, \ldots, M$. Now we can formulate the least square framework with residual $\mathbf{r}=\mathbf{b}-\mathbf{A s}$, such that;

$$
\hat{\mathbf{s}}=\underset{\mathbf{s}}{\operatorname{argmin}}\left\{\mathbf{r}^{T} \mathbf{r}\right\} .
$$

Then, a linear least square estimator for (15) can be directly obtained by using the closed-form solution [15]: $\hat{\mathbf{s}}=\left(\mathbf{A}^{T} \mathbf{A}\right)^{-1} \mathbf{A}^{T} \mathbf{b}$. For open and noncongested indoor environments with small or medium scale networks, LLS approach can yield acceptable position estimates, however, it may become erroneous and computationally challenging for complex indoor environments and large scale wireless networks. 


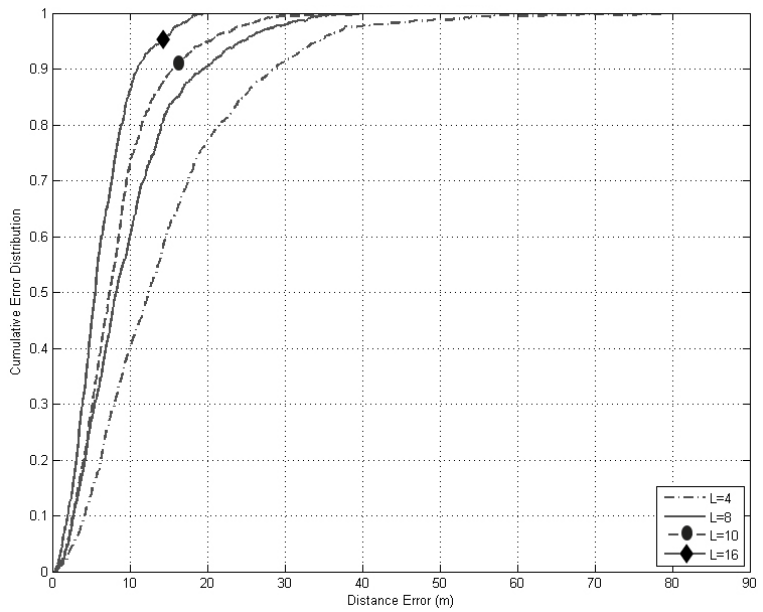

(a)

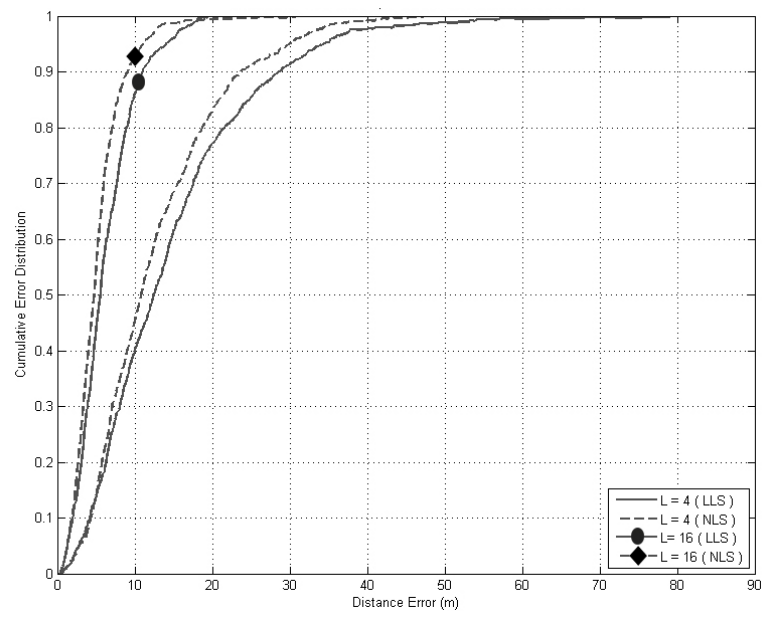

(c)

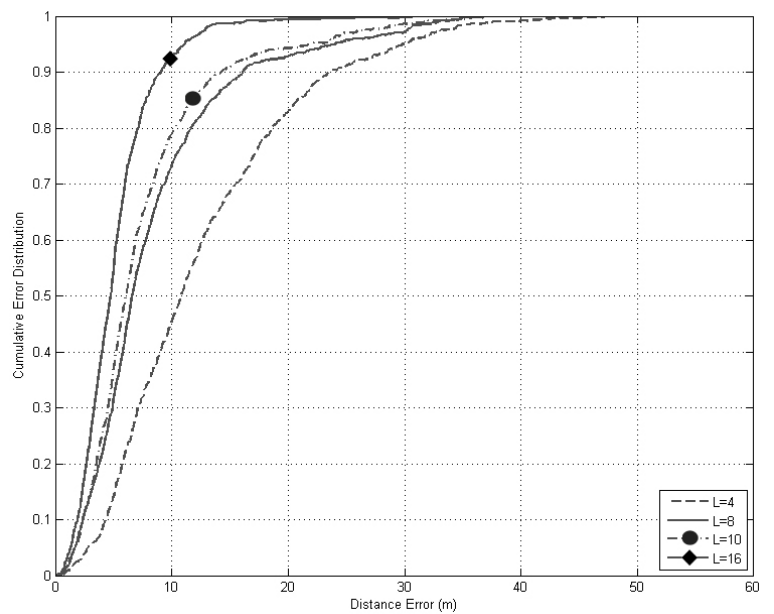

(b)

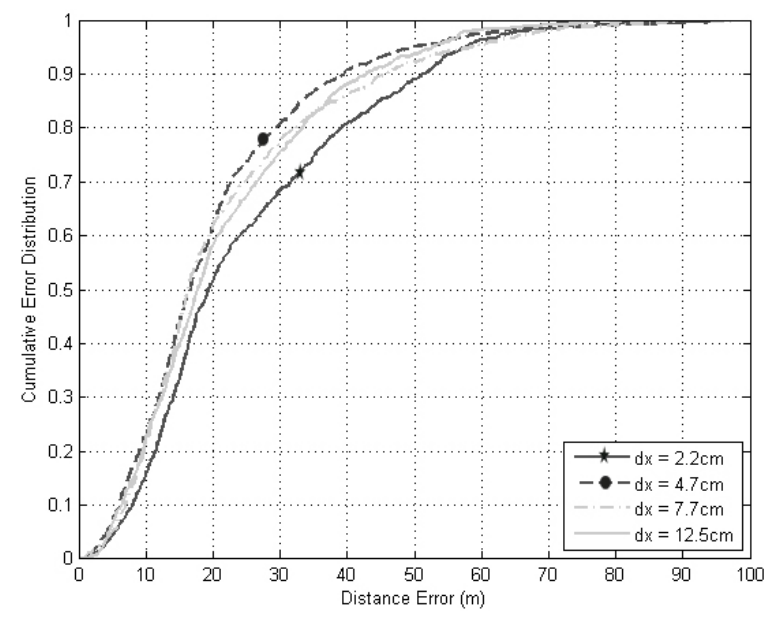

(d)

Figure 4. Localization precision comparison: (a) for LLS lateration (b) for NLS lateration (c) LLS and NLS (d) Inter-element space vs precision (for correlated channels with $L=2$

\section{NuMERICAL EXAMPLES}

In this section, we study the effect of using multi-element antenna arrays at the mobile receivers for positioning improvement through numerical simulations. The accuracy of the positioning engine is defined as the Euclidean distance between the estimated location and actual location of MS for a given localization attempt. The cumulative distribution function (CDF) of location accuracy is used for measuring the precision of the localization system [16].

In the first example, we simulate a Rayleigh fading environment where channels are i.i.d circularly complex Gaussian distributed with zero mean and variance 2 . All the transmitters (i.e. WAPs) transmit equivalent BPSK signal of the sequence '01111110' with $2.4 \mathrm{GHz}$ carrier frequency, and receiver frontend SNR is $10 \mathrm{~dB}$. We simulate MRC diversity reception systems with $L=4,8,10$ and 16 , and positioning estimates are obtained through LLS lateration. It can be seen from Fig. 4a that the precision of the estimates increases as the number of elements on the array increases.

In the second example, we simulate the same wireless system configuration but replacing the positioning algorithm with NLS. The outputs shows a similar behaviour to LLS but with improved performance as depicted in Fig. 4b. Fig. 4c illustrates the performance improvement of NLS compared to LLS. Finally, we take the correlation between antenna elements into consideration and simulate for a $L=2 \mathrm{MRC}$ system with various inter-element spaces, with NLS approach. It can be seen from Fig. 4d that the localization performance is relatively high when inter spacing between two antenna is $4.7 \mathrm{~cm}$ at which variance in (11) is minimum.

\section{CONCLUSION}

In this work, we studied the impact of using antenna arrays at receiving stations on improving the accuracy of the location estimates which is typically degraded due to multipath fading in indoor environments with single antenna systems. We simulated a fixed 802.11 indoor multipath propagation environment 
and MRC diversity reception systems. We further adapted two widely used lateration based algorithms to validate our proposal. We theoretically showed the reduction of received signal strength temporal fluctuations with the deployment of multi-element antennas. Simulation results confirmed that increase in the number of elements on the array leads to considerable enhancement for localization accuracy.

\section{REFERENCES}

[1] K. Pahlavan, X. Li, and J. Makela, "Indoor geolocation science and technology," IEEE Communications Magazine, vol. 40, no. 2, pp. 112 -118 , Feb. 2002.

[2] G. Sun, J. Chen, W. Guo, and K. Liu, "Signal processing techniques in network-aided positioning: A survey of state-of-the-art positioning designs,” IEEE Signal Processing Magazine, vol. 22, no. 4, pp. 12 - 23, July 2005

[3] A. Sayed, A. Tarighat, and N. Khajehnouri, "Network-based wireless location: Challenges faced in developing techniques for accurate wireless location information," IEEE Signal Processing Magazine, vol. 22, no. 4, pp. $24-40$, July 2005.

[4] P. Bahl and V. Padmanabhan, "RADAR: An in-building RF-based user location and tracking system," in Proc. 19th Annual Joint Conference of the IEEE Computer and Communications Societies, vol. 2, Mar. 2000, pp. $775-784$.

[5] A. de Toledo and A. Turkmani, "Propagation into and within buildings at 900, 1800 and $2300 \mathrm{MHz}$," in Proc. 42nd IEEE Vehicular Technology Conference, May 1992, pp. $633-636$.

[6] D.-S. Shiu, G. Foschini, M. Gans, and J. Kahn, "Fading correlation and its effect on the capacity of multielement antenna systems," IEEE Transactions on Communications, vol. 48, no. 3, pp. $502-513$, Mar. 2000.
[7] J. Yang, Y. Chen, V. Lawrence, and V. Swaminathan, "Robust wireless localization to attacks on access points," in Proc. IEEE Sarnoff Symposium, 2009, Apr. 2009, pp. $1-5$.

[8] H. Hashemi, "The indoor radio propagation channel," Proceedings of the IEEE, vol. 81, no. 7, pp. 943 -968, July 1993.

[9] P. Pahlavan and A. H. Levesque, Wireless Information Networks, 2nd ed. Hoboken, NJ, USA: John Wiley \& Sons, 2005.

[10] V. Erceg, L. Schumacher, P. Kyritsi, D. Baum, A. Molisch, and A. Gorokhov, Indoor MIMO LAN Channel Models, IEEE Stand. 802.11 03/161r0, March 2003.

[11] T. Rappaport, Wireless Communications: Principles and Practice, 2nd ed. Prentice Hall, 2002.

[12] R. Adve, "Diversity reception," Toronto, Ontario, Canada. [Online]. Available: http://www.comm.utoronto.ca/ rsadve/Notes/ DiversityReceive.pdf

[13] C. Oestges, B. Clerckx, D. Vanhoenacker-Janvier, and A. Paulraj, "Impact of fading correlations on MIMO communication systems in geometry-based statistical channel models," IEEE Transactions on Wireless Communications, vol. 4, no. 3, pp. 1112 - 1120, May 2005.

[14] D. Bertsimas, "Optimization methods - line searches and newton's method," Cambridge, MA 02139-4307. [Online]. Available: http://ocw.mit.edu/courses/sloan-school-of-management/ 15-093j-optimization-methods-fall-2009/lecture-notes/MIT15 \_093J F09 \_lec19.pdf

[15] J. Yang and Y. Chen, "Indoor localization using improved RSS-based lateration methods," in IEEE Global Telecommunications Conference, Dec. 2009, pp. 1-6.

[16] K. Kleisouris, Y. Chen, J. Yang, and R. Martin, "The impact of using multiple antennas on wireless localization," in 5th Annual IEEE Communications Society Conference on Sensor, Mesh and Ad Hoc Communications and Networks, June 2008, pp. 55 -63. 